\title{
As opções terapêuticas para os aneurismas fusiformes
}

\author{
Maria Luana Carvalho Viegas ${ }^{1}$, Daniella Brito Rodrigues², Saulo Araújo Teixeira ${ }^{3}$, \\ Eberval Gadelha Figueiredo ${ }^{4}$, José Guilherme Caldas ${ }^{5}$, Manoel Jacobsen Teixeira ${ }^{6}$ \\ Divisão de Neurocirurgia da Faculdade de Medicina da Universidade de São Paulo, São Paulo, SP, Brasil.
}

\section{RESUMO}

Os aneurismas fusiformes são pouco frequentes e geralmente não estão associados a fatores de risco específicos. Ocorrem principalmente nas artérias cerebrais, por meio de uma dilatação circunferencial na parede da artéria, garantindo seu aspecto morfológico típico: ausência de colo aneurismático. Por essa razão, não podem ser usualmente clipados ou embolizados, necessitando de métodos alternativos de tratamento que representam um desafio para os neurocirurgiões. As alternativas cirúrgicas estão associadas a um melhor prognóstico e incluem as técnicas de revascularização com o uso de bypass, a oclusão da artéria aferente (PAO, sigla em inglês) e "wrapping" ou clip-wrap. Métodos endovasculares, como o uso de stents, representam uma opção para restauração mais fisiológica do fluxo sanguíneo, embora necessitem de mais estudos para confirmar a eficácia.

\section{PALAVRAS-CHAVE}

Aneurismas intracranianos/cirurgia, aneurisma dissecante, procedimentos cirúrgicos operatórios.

\section{ABSTRACT}

The terapeutic options for fusiform aneurysms

The fusiform aneurysms are uncommon and usually are not associated with specific risk factors. They occur especially in the cerebral arteries, around the circumferential dilatation of the cerebral artery wall, ensuring their morphological characteristic: absence of aneurysm neck. For this reason, can not usually be clipped or embolized, requiring alternative treatment methods that represent a challenge for neurosurgeons. The surgeries alternatives are associated with better prognostic and revascularization techniques include the use of bypass, parent artery occlusion (PAO) and wrapping or clip-wrap. The endovascular method represent an option for restoration of the physiologic blood flow; however it's necessary more studies for confirm the technical efficacy.

\section{KEYWORDS}

Intracranial aneurysms/surgery, aneurysm dissecting, surgical procedures operative.

Residente de Neurocirurgia do Hospital Ophir Loyola (HOL), Belém, PA, Brasil.

2 Discente do curso de Medicina da Universidade do Estado do Pará (UEPA), Belém, PA, Brasil.

3 Discente do curso de Medicina da Universidade Federal do Ceará (UFC), Fortaleza, PA, Brasil.

4 Supervisor da Divisão de Clínica Neurocirúrgica e chefe do Grupo de Neurocirurgia Vascular do Hospital das Clínicas da Faculdade de Medicina da Universidade de São Paulo (HCFMUSP), São Paulo, SP, Brasil.

5 Chefe do Departamento de Radiologia Intervencionista do HCFMUSP, São Paulo, SP, Brasil.

6 Professor titular da Divisão de Clínica Neurocirúrgica do Hospital das Clínicas e professor titular da Disciplina de Neurocirurgia da USP, São Paulo, SP, Brasil. 


\section{Introdução}

Aneurismas intracranianos são dilatações patológicas dos vasos que podem ter uma parede anormalmente fraca e propensa à ruptura, causando hemorragia subaracnóidea (HSA) e, às vezes, hemorragia intraparenquimatosa. A incidência de HSA aneurismática é estimada em 10-11/100000 na população ocidental, apresentando taxas de mortalidade que se aproximam de 30-40\%, com elevada morbidade dos sobreviventes. A maior parte dos aneurismas intracranianos está localizada nas artérias cerebrais, apresentando-se de diversas morfologias, sendo os mais comuns os saculares, portadores de um colo passível de clipagem ou embolização, em menor frequência, porém, de difícil manejo terapêutico, existem os aneurismas fusiformes. ${ }^{1}$

Diferentes termos têm sido usados para descrever aneurismas que não podem ser diretamente clipados ou embolizados. Alguns desses, como "aneurisma dissecante”, referem-se ao provável mecanismo etiológico, enquanto outros como "dolichoectáticos" e "fusiformes" dizem respeito à descrição morfológica. ${ }^{2}$ Os aneurismas fusiformes são caracterizados por uma dilatação e estiramento da artéria e podem ser agudos ou crônicos com base na evolução clínica. ${ }^{3}$

Existe um espectro grande dos aneurismas dolichoectáticos que varia desde pequenas dilatações aneurismáticas fusiformes de um único vaso até aneurismas gigantes cheios de trombose (aneurismas gigantes de serpentina). Esses aneurismas, principalmente os parcialmente trombosados, muitas vezes produzem sintomas de compressão neural, por embolização distal ou causando hemorragia subaracnóidea (HSA). ${ }^{4}$

Aneurismas fusiformes são lesões de difícil tratamento e frequentemente necessitam de técnicas alternativas de tratamento, incluindo anastomoses extraintracranial ou técnicas de “clip-wrap". Para uma técnica adequada, a artéria deve ser circunferencialmente dissecada. ${ }^{5-7}$

\section{Aneurismas fusiformes}

Os aneurismas fusiformes representam 1\% dos aneurismas cerebrais e usualmente ocorrem na artéria vertebrobasilar, artéria carótida interna e artéria cerebral média. ${ }^{8}$ São mais frequentes na população pediátrica em comparação com a frequência em adultos. ${ }^{1,9}$

A etiologia é diversa e a aterosclerose pode ser a causa subjacente, mas nem sempre está presente. Estudos histológicos têm demonstrado defeitos congênitos na lâmina elástica interna da artéria e displasia segmentar que pode ocorrer em crianças. Estão associados com lúpus eritematoso sistêmico, vasculites e outras doenças do colágeno. Infecções (aneurismas micóticos, por exemplo), malformações vasculares (MAVs), dissecção idiopática, e, muito raramente, invasão neoplásica da parede arterial também podem estar implicadas. ${ }^{10}$ Drake et al. apud Saito ${ }^{9}$ et al. e Gobin ${ }^{10}$ et al., em uma revisão de 120 casos de pacientes com aneurismas fusiformes, não encontraram causa específica para 111 deles, doenças reumatológicas e cromossomopatias foram identificadas nos demais doentes.

Esse tipo de aneurisma ocorre com menor frequência que os saculares, e as características demográficas, a apresentação clínica e a história natural são distintas. Pacientes com aneurismas fusiformes podem se apresentar com hemorragia subaracnóidea (HSA), déficits neurológicos focais por efeito de massa, isquemia, ou sintomas inespecíficos. ${ }^{2}$

Em função de não apresentar um colo definido que possibilite a clipagem com preservação do vaso aferente, são particularmente difíceis de tratar cirurgicamente. Frequentemente, a artéria é envolvida circunferencialmente por uma dilatação aneurismática fusiforme ou completamente perdida em uma maciça trombose com ramos distais que partem do centro do aneurisma. Eliminando-se os riscos de hemorragia ou compressão sem comprometer a circulação cerebral distal no território vascular, muitas vezes os tratamentos disponíveis requerem técnicas não usuais e de difícil execução. ${ }^{4}$

\section{Classificação}

De acordo com a evolução clínica, os aneurismas dissecantes representam a modalidade aguda e desenvolvem um desfecho desfavorável devido à hemorragia subaracnóidea ou isquemia cerebral. O tipo crônico raramente apresenta sangramento e com mais frequência pode causar acidente vascular cerebral ou ataque isquêmico transitório por meio de vários mecanismos: distensão e distorção nos pontos de ramificação e nas artérias adjacentes, vasoespasmo, mecanismos hemodinâmicos, por trombose no aneurisma fusiforme, seja por embolização distal ou por oclusão dos ramos da artéria de origem. ${ }^{3}$

Com base em estudos de casos e autopsias, Mizutani et al. ${ }^{11}$ determinaram uma classificação para os aneurismas fusiformes em quatro tipos principais: 1) O tipo I representa os aneurismas dissecantes clássicos, são formados durante um processo agudo, o qual apresenta com frequência hemorragia subaracnóidea (HSA), evoluindo com prognóstico sombrio e probabilidade elevada de ressangramento, e histologicamente não há espessamento da camada íntima da artéria. 2) Os 
de tipo II apresentam ectasia segmentar, geralmente são assintomáticos e têm associação com patologias cardiovasculares ou história prévia de neurocirurgias. Não há presença de trombos intraluminais e a camada intimal demonstra um espessamento moderado. 3) A característica mais típica do aneurisma tipo III é o aspecto angiográfico de um aneurisma fusiforme tortuante, que contrasta irregularmente ao exame devido à presença de trombos luminais. 4) Os aneurismas tipo IV aparecem na angiografia semelhantes aos saculares, porém diferindo desses pela ausência de um colo específico a partir da artéria aferente. ${ }^{11}$

São classificados em simples quando a dilatação aneurismática não inclui ramificações da artéria dilatada, e os complexos acontecem quando há ramos próximos ao aneurisma, fator que deve ser levado em consideração ao se planejar as técnicas de tratamento. ${ }^{12}$

\section{Diagnóstico e estratégias pré-operatórias}

A análise detalhada de métodos diagnósticos pré-operatórios é essencial para se prevenir falhas do tratamento convencional. A tomografia computadorizada (TC) e a angiotomografia (ATC) permitem melhor identificação de calcificações no colo do aneurisma. Angiorressonância magnética (ARM) é mais adequada para estimar a extensão da trombose no interior do aneurisma. Angiografia com subtração digital permite a visualização do aneurisma sob ângulos diferentes, possibilitando uma delimitação anatômica mais adequada, incluindo as colaterais aferentes e eferentes, bem como as ramificações e perfurantes. Angiografias seletivas do aneurisma, embora raramente realizadas, podem ser adequadas. Reconstruções tridimensionais feitas pelas técnicas de ATC ou ARM contribuem significativamente para a avaliação pré-operatória do aneurisma, especialmente no que se refere à visualização dos ramos proximais, distais ou transmurais. ${ }^{13}$

O teste oclusivo por balão (BTO) é um importante teste provocativo quando inclui a oclusão da artéria principal para o tratamento de aneurismas complexos. O BTO avalia a circulação colateral cerebral em situações específicas, guiando os procedimentos de revascularização e estimando riscos intra- e pós-operatórios que contraindicariam a cirurgia. O BTO deve ser realizado somente na ausência de condições patológicas concomitantes, como presença de vasoespasmo sintomático, hemorragias subaracnóideas, pacientes com artérias comunicantes de pequeno calibre e presença de múltiplos aneurismas. ${ }^{14-16}$

Geralmente, há obstrução da artéria carótida interna, com hipotensão induzida, por cerca de 20 minutos. Outras modalidades de imagem podem ser auxiliares da BTO e incluem SPECT, tomografia de perfusão cerebral, Doppler transcraniano, EEG contínuo e avaliação neurológica no momento do exame. Em geral, essas modalidades têm melhorado o valor preditivo da BTO por documentarem o fluxo sanguíneo cerebral durante a oclusão. ${ }^{17}$

Os métodos de revascularização devem ser orientados por monitorização eletrofisiológica contínua, incluindo o EEG e o de potenciais evocados. $\mathrm{O}$ uso de microDoppler ajuda a avaliar a eficácia da clipagem e o fluxo sanguíneo proximal, a artéria ocluída, por meio do bypass e distalmente à anastomose. No entanto, a utilização da angiografia intraoperatória continua a ser um método de eficácia imediata no momento da reconstrução. Restrições pertinentes ao campo cirúrgico e a presença de falso-negativos continuam a ser uma limitação dessa tecnologia. ${ }^{12}$

\section{Tratamento}

O tratamento dos aneurismas cerebrais exibiu melhorias significativas nos últimos anos, com o advento das técnicas de microcirurgia em 1960 e o desenvolvimento de métodos endovasculares nos anos de 1980. No entanto, os aneurismas fusiformes representavam um desafio significante para os neurocirurgiões porque nenhum desses métodos era eficaz para o manejo seguro desses aneurismas. ${ }^{18} \mathrm{O}$ tratamento dos fusiformes requer modalidades alternativas como bypass, wrapping e clip-wrapping. ${ }^{2,710,19}$

\section{Estratégias cirúrgicas}

\section{Técnicas de revascularização}

O tratamento dos aneurismas cerebrais fusiformes é um assunto controverso. Para prevenir complicações isquêmicas após excisão ou trapping, estudos pioneiros ${ }^{20,21}$ descreveram técnicas de anastomoses microvasculares para restaurar a perfusão da porção do vaso distal à artéria ocluída. Nessas o aneurisma é excisado e as porções proximal e distal do vaso são anastomosadas. Em 1986, Chang et al. ${ }^{21}$ relatam um caso de aneurisma fusiforme gigante de cerebral posterior, tratado com sucesso por excisão do aneurisma e anastomose terminoterminal, realizando um procedimento seguro que se mostrou superior a outras opções até então existentes.

Em estudos recentes, a cirurgia de bypass extracraniano-intracraniano (EC-IC) tem-se tornado parte de uma estratégia multidisciplinar altamente especializada para a 
terapia de aneurismas complexos. ${ }^{22,23} \mathrm{~A}$ escolha da estratégia de revascularização deve ser individualizada para o paciente, dependendo de fatores do aneurisma, tais como o tamanho (maior ou menor que $2 \mathrm{~mm}$ ), a localização do vaso que participará do procedimento (proximal/distal), a possibilidade de vias colaterais e a avaliação do material do enxerto e do fluxo sanguíneo no aneurisma. ${ }^{13,24}$

Os vários procedimentos com bypass podem ser classificados em quatro tipos: Tipo 1 envolve a interposição de um enxerto, frequentemente da veia safena, partindo da artéria aferente para anastomose com a porção distal ao aneurisma. Tipo 2 de bypass refere-se a uma interposição de enxerto da veia safena entre a artéria carótida externa e um grande ramo intracraniano como a artéria cerebral média (ACM) e a artéria cerebral posterior (ACP). Tipo 3 consiste em utilizar uma artéria do couro cabeludo, como a artéria temporal superficial, como vaso doador, que seria anastomosado a um ramo distal - artéria cerebelar posteroinferior (PICA), artéria cerebral média (ACM) ou artéria cerebelar superior (ACS). O tipo 4 envolve a anastomose entre duas artérias adjacentes, in situ, que pode ser laterolateral, terminoterminal ou por meio da conexão entre ramos (anastomoses terminolaterais). ${ }^{25,26}$

Os intracranianos-intracranianos bypass são interessantes por não incluírem artérias extracranianas na anastomose, reconstruindo a circulação cerebral com a vascularização intracraniana normal, assegurando uma correspondência de diâmetro entre a artéria doadora e a receptora. Esse tipo de reanastomose requer que as artérias participantes estejam em paralelo e/ou em estreita proximidade. Apesar de seu maior desafio técnico, estudos recentes relatam que bypass IC-IC apresenta melhores resultados quando comparado com EC-IC em termos de taxas de perviedade do enxerto, de obliteração do aneurisma e evolução neurológica. ${ }^{25-27}$

No final da década de 1990, Tulleken desenvolveu a técnica de excimer laser-assisted non-occlusive anastomosis (Elana). Esse procedimento estabelece um bypass venoso lateroterminal usando uma técnica de anastomose não oclusiva com o laser excimer de $308 \mathrm{~nm}$. As maiores vantagens da técnica de Elana são de impedir a oclusão temporária do vaso receptor, não interrompendo o fluxo sanguíneo para o cérebro, não expor o vaso receptor e permitir anastomoses mais proximais, incluindo os grandes vasos intracranianos como a artéria carótida interna (ACI)..$^{28,29}$

A aplicação dessa nova técnica não encurta o tempo cirúrgico e de fato pode até aumentá-lo devido à inserção de um anel de platina no vaso receptor. Nenhum estudo ainda concluiu se os pacientes tratados com Elana apresentam maior benefício funcional a longo prazo. Entretanto, Elana como bypass de alto fluxo é uma alternativa segura às técnicas de bypass convencionais, sem alterá-las de forma significativa ou interferir em seus resultados. ${ }^{13,29}$
A avaliação pós-operatória da permeabilidade do bypass é feita com a utilização de angiografia com subtração digital. Outras opções são a tomografia computadorizada e a angiorressonância, sendo os métodos não invasivos de imagem ainda pouco confiáveis devido aos pequenos diâmetros de anastomoses microvasculares. ${ }^{30}$

\section{Oclusão da artéria aferente}

Para os adeptos da revascularização seletiva, a oclusão da artéria aferente do aneurisma (PAO) é uma opção de tratamento se o fluxo da circulação colateral demonstrado pela BTO não for suficiente para o reencher. ${ }^{9,31,32} \mathrm{PAO}$ tende a ser mais eficaz para os aneurismas dos segmentos petroso e cavernoso da artéria carótida interna, devido ao pequeno número de ramificações e ao delgado calibre destas. ${ }^{13}$

Para alguns aneurismas complexos da artéria cerebral média $(\mathrm{ACM})$ e da porção distal de artéria cerebral anterior (ACA), a oclusão proximal e distal do vaso (trapping) pode ser preferível, uma vez que fornece isolamento completo a partir da circulação, evita o risco de hemorragia por enchimento retrógrado, além de permitir a descompressão imediata do aneurisma para aliviar o efeito de massa. ${ }^{33}$

Em circunstâncias que o bypass não é necessário e a PAO é suficiente para o tratamento do aneurisma, meios endovasculares de oclusão, usando balões intra-arteriais ou embolização, podem ser realizados. ${ }^{34}$ Com efeito, a oclusão endovascular permite obstruir a artéria aferente próximo à saída do aneurisma, evitando-se assim a formação de uma longa coluna de trombose intraluminal e potenciais complicações tromboembólicas relacionadas à dissecção do colo do aneurisma. ${ }^{10,31}$

No entanto, em circunstâncias em que o bypass e PAO são planejados concomitantemente, é possível a oclusão cirúrgica da artéria aferente após a verificação de permeabilidade do bypass. Entretanto, se o isolamento arterial não é possível durante a realização do bypass, meios endovasculares de oclusão podem ser considerados. $\mathrm{O}$ vaso deve ser ocluído próximo ao colo aneurismático para diminuir a probabilidade de trombose e embolia. ${ }^{13}$

Mesmo na presença de um bypass patente, com um procedimento realizado após a avaliação precisa da reserva vascular cerebral, complicações isquêmicas podem ocorrer após PAO e são difíceis de serem previstas. A recidiva pode acontecer em até $10 \%$ dos casos, e o crescimento do aneurisma e sua ruptura têm sido relatados após PAO, provavelmente por alterações do fluxo sanguíneo cerebral e da dinâmica do polígono de Willis; uma alternativa seria a realização concomitante de bypass endovascular. ${ }^{35}$ 


\section{Clip-wrap ou wrapping}

As técnicas de wrapping ou clip-wrap são métodos seguros e estão associados a baixo risco de complicações agudas ou mortalidade no pós-operatório. Podem prevenir ressangramento e crescimento dessas lesões e representam uma mudança na história natural desses complexos aneurismas. ${ }^{5,736}$

As técnicas de wrapping e clip-wrap utilizam uma "malha" de material sintético, que inicialmente era de dracon ou silastick, para o tratamento dos aneurismas fusiformes. A artéria aferente deve ser adequadamente dissecada, e duas folhas da "malha" envolvem circunferencialmente o aneurisma, evitando o aprisionamento dos nervos cranianos e de ramificações da artéria. $\mathrm{O}$ material deve ser semitransparente e se adapta facilmente ao calibre do vaso. Depois que a artéria é adequadamente envolvida por "wrap", o clip é inserido para fixar as extremidades das malhas. A força de preensão e a adaptação do material ao vaso devem ser adequadas para evitar deslizamento, compressão e prevenir complicações tardias. ${ }^{37,38}$

Um estudo experimental conduzido por Herrera et al. ${ }^{39}$ utilizou a técnica de clip-wrap em ratos para avaliar as alterações histológicas a longo prazo, e os resultados indicaram que a fibrina produzia inflamação aguda nas primeiras duas semanas, que desaparecia após dois meses, persistindo apenas uma inflamação da adventícia. Biobond causa inflamação crônica, necrose da túnica média e espessamento da parede arterial da túnica média e adventícia. Bemsheet produz inflamação crônica, fibrose progressiva e granuloma. Tecido conjuntivo aumenta na adventícia, mas maiores mudanças são observadas na túnica média. As fibras Bemsheet permaneceram inalteradamente aderidas à parede arterial. Estenoses arteriais não foram observadas nesse estudo, mas os resultados sugerem que a cola de cianoacrilato pode causar lesões arteriais oclusivas..$^{39}$ Estudos recentes, entretanto, apontam que os riscos de complicações tardias da técnica como formação de granulomas ou aracnoidites são baixos. ${ }^{7,40}$

Existe um método alternativo da técnica de clip-wrap para os aneurismas fusiformes que promove um suporte estrutural para a reconstrução da artéria ou aneurisma residual por meio da utilização de um sling Gore-Tex que permite a reconstituição da parede dos vasos, diminuindo a possibilidade de neoformação do aneurisma, uma vez que nas demais técnicas a parede permanece suscetível. ${ }^{41}$

\section{Estratégias endovasculares}

\section{Embolização}

A técnica de embolização provou ser efetiva no tratamento de grande parte dos aneurismas intracranianos, mas aneurismas mais complexos, como os fusiformes, são tecnicamente desafiadores. Ainda são mais suscetíveis a recidivas que ocorrem em $20-30 \%$ e resultam em necessidade de retratamento em $10-12 \%$ dos casos. ${ }^{42}$

Por essas razões, as técnicas de embolização não são adequadas para o tratamento dos aneurismas fusiformes, a formação de trombos pode se estender pela artéria ocluída, causando isquemia, ou pode acontecer a dissolução do trombo, quando a técnica se torna inútil. Entretanto, pode se apresentar como opção para prevenir as consequências sintomáticas do vasoespasmo, enquanto se aguarda a realização da cirurgia, pois nenhum dos casos tratados por esse método apresenta resultados satisfatórios a longo prazo. ${ }^{43}$

\section{Stents}

Os avanços recentes permitiram a utilização de stents para o tratamento endovascular dos aneurismas fusiformes ${ }^{41,44}$ Chiaradio et al. ${ }^{18}$ descreveram, em 2002, o sucesso da introdução de um stent enxerto para o tratamento de um aneurisma fusiforme tipo I da artéria cerebelar posteroinferior. Crowley et al.${ }^{43}$ descreveram, em 2009, os bons resultados obtidos com a inserção de um stent em aneurisma fusiforme da artéria basilar, provocando trombose do aneurisma, diminuindo o efeito de massa sobre o tronco cerebral e mantendo níveis de perfusão adequados. Luo et al..$^{45}$ utilizaram stents para o tratamento de aneurismas fusiformes do segmento P2 da artéria cerebral posterior, com bons resultados pós-operatórios.

$\mathrm{O}$ uso de stents de enxerto na vascularização intracraniana pode ter efeitos deletérios sobre o paciente devido à presença de vasos colaterais da artéria principal, mas as técnicas de subtração de imagem e métodos ultrassonográficos disponíveis hoje permitem a determinação dos locais de óstio, garantindo uma inserção mais adequada para o procedimento. A vantagem óbvia do uso de stents de enxerto é que o lúmen do aneurisma é fechado no momento em que o stent se adapta à parede arterial. ${ }^{18}$

A utilização de stents foi proposta em meados da década de 1990, inicialmente para aneurismas fusiformes e mais recentemente para apoiar o colo dos aneurismas saculares e também para prevenir recorrência. Um stent inserido na região de estagnação e trombose da artéria reduz o fluxo no interior do aneurisma, excluindo-o do fluxo sanguíneo cerebral. ${ }^{42,43}$

Novos stents recentemente foram aprovados para uso clínico, prometem ser flexíveis o suficiente para se adaptarem aos vasos intracranianos e são constituídos com fios de tecido de nitinol de baixa porosidade para reter o fluxo na artéria de origem e não permitir o desvio de sangue para o saco aneurismático. O stent SILK (Balt Extrusion, Montmorency, France) foi aprovado em 2007 e, a fim de avaliar a segurança e a aplicabilidade do grupo SDF (Stents de seda do qual o SLIK faz parte), 
foi realizado um estudo multicêntrico cujos resultados demonstram dificuldades processuais no SFD..$^{42}$ Mesmo em equipes familiarizadas com a técnica, as taxas de mortalidade e complicações do método continuam superiores aos métodos tradicionais como os de wrapping. ${ }^{46}$

Complicações como tromboembolismo, com consequente acidente vascular encefálico isquêmico de outras artérias, estenose ou oclusão total da artéria podem ocorrer. ${ }^{41}$ Por outro lado, o uso de profilaxia antitrombótica intensiva pode estar associado com hemorragias pós-procedimento..$^{42}$ Entretanto, alguns autores acreditam que a utilização de um stent represente a opção mais fisiológica para a reconstrução da artéria nos aneurismas fusiformes. ${ }^{41}$

A associação conjunta entre embolização seguida pela passagem de um stent para ocluir o vaso tem se mostrado como alternativa mais eficaz no tratamento endovascular dos aneurismas fusiformes, entretanto a necessidade de anticoagulação plena e a possibilidade de hemorragia subaracnóidea e/ou ressangramento do aneurisma deixa essa opção ainda pouco segura, ${ }^{46}$ embora o tratamento de um aneurisma fusiforme da artéria cerebral média tenha obtido bons resultados com essa associação. ${ }^{47} \mathrm{~A}$ associação conjunta de um stent com um polímero líquido sintético para a oclusão do vaso já foi descrita na literatura, entretanto, o número restrito de pacientes submetidos ao procedimento e a eficácia não comprovada do método não implicam perspectivas para essa forma de manejo. ${ }^{48}$

\section{Conclusão}

Estudos indicam maior eficácia com o uso de técnicas cirúrgicas para o tratamento dos aneurismas complexos como os fusiformes. Particularmente o uso de bypass é promissor e está associado a menores complicações pós-operatórias que a $\mathrm{PAO}$, tais como isquemia e ressangramento. Clip-wrap é eficaz, mas a longo prazo se relaciona ao recrescimento do aneurisma. A escolha da opção por métodos endovasculares ou intervencionistas deve levar em consideração a presença de sintomas, os riscos de crescimento, o ressangramento, a isquemia e outras complicações, tendo em vista sempre um planejamento e discussão feitos por equipe multidisciplinar de neurocirurgiões e radiologistas intervencionistas, $\mathrm{e}$ ainda a opção do paciente, depois de apresentadas as vantagens e desvantagens de cada técnica.

\section{Conflitos de interesse}

Os autores declaram não haver conflitos de interesse.

\section{Referências}

1. Frösen J, Tulamo R, Paetau A, Laaksamo E, Korja $M$, Laakso A, et al. Saccular intracranial aneurysm: pathology and mechanisms. Acta Neuropathol. 2012;123(6):773-86.

2. Coert BA, Chang SD, Do HM, Marks MP, Steinberg GK. Surgical and endovascular management of symptomatic posterior circulation fusiform aneurysms. J Neurosurg. 2007;106(5):855-65.

3. Cappellari M, Tomelleri G, Piovan E, Bovi P, Moretto G, Gulli G. Chronic fusiform aneurysm evolving into giant aneurysm in the basilar artery. Neurol Sci. 2012;33(1):111-5.

4. Anson JA, Lawton MT, Spetzler RF. Characteristics and surgical treatment of dolichoectatic and fusiform aneurysms. J Neurosurg. 1996;84(2):185-93.

5. Figueiredo EG, Foroni L, Monaco BA, Gomes $M Q$, Sterman Neto $H$, Teixeira MJ. The clip-wrap technique in the treatment of intracranial unclippable aneurysms. Arq Neuropsiquiatr. 2010;68(1):115-8.

6. Weir B. Unruptured aneurysms. J Neurosurg. 2002;97(5):1011-2.

7. Deshmukh VR, Kakarla UK, Figueiredo EG, Zabramski JM, Spetzler RF. Long-term clinical and angiographic follow-up of unclippable wrapped intracranial aneurysms. Neurosurgery. 2006;58(3):434-42.

8. Kimura T, Nishimura K, Fukaya S, Morita A. Fusiform aneurysm of the anterior communicating artery treated by vascular reconstruction: case report. Neurosurgery. 2010;66(5):E1025-6.

9. Saito $\mathrm{H}$, Ogasawara K, Kubo $\mathrm{Y}$, Tomitsuka N, Ogawa A. Treatment of ruptured fusiform aneurysm in the posterior cerebral artery with posterior cerebral arterysuperior cerebellar artery anastomosis combined with parent artery occlusion: case report. Surg Neurol. 2006;65(6):621-4.

10. Gobin YP, Viñuela F, Gurian JH, Guglielmi G, Duckwiler GR, Massoud TF, et al. Treatment of large and giant fusiform intracranial aneurysms with Guglielmi detachable coils. J Neurosurg. 1996;84(1):55-62.

11. Mizutani T, Miki Y, Kojima H, Suzuki H. Proposed classification of nonatherosclerotic cerebral fusiform and dissecting aneurysms. Neurosurgery. 1999;45(2):253-9.

12. Pritz MB. Cerebral aneurysm classification based on angioarchitecture. J Stroke Cerebrovasc Dis. 2011;20(2):162-7.

13. McLaughlin N, Gonzalez N, Martin NA. Surgical strategies for aneurysms deemed unclippable and uncoilable. Neurochirurgie. 2012;58(2-3):199-205.

14. Tarr RW, Jungreis CA, Horton JA, Pentheny S, Sekhar LN, Sen C, et al. Complications of preoperative balloon test occlusion of the internal carotid arteries: experience in 300 cases. Skull Base Surg. 1991;1(4):240-4.

15. Mathis JM, Barr JD, Jungreis CA, Yonas H, Sekhar LN, Vincent $D$, et al. Temporary balloon test occlusion of the internal carotid artery: experience in 500 cases. AJNR Am J Neuroradiol. 1995;16(4):749-54.

16. Dare AO, Chaloupka JC, Putman CM, Fayad PB, Awad IA. Failure of the hypotensive provocative test during temporary balloon test occlusion of the internal carotid artery to predict delayed hemodynamic ischemia after therapeutic carotid occlusion. Surg Neurol. 199;50(2):147-55.

17. Abud DG, Spelle L, Piotin M, Mounayer C, Vanzin JR, Moret $\mathrm{J}$. Venous phase timing during balloon test occlusion as a criterion for permanent internal carotid artery sacrifice. AJNR Am J Neuroradiol. 2005;26(10):2602-9. 
18. Chiaradio JC, Guzman L, Padilla L, Chiaradio MP. Intravascular graft stent treatment of a ruptured fusiform dissecting aneurysm of the intracranial vertebral artery: technical case report. Neurosurgery. 2002;50(1):213-6.

19. Atalay B, Altinors N, Yilmaz C, Caner H, Ozger O. Fusiform aneurysm of the superior cerebellar artery: short review article. Acta Neurochir (Wien). 2007;149(3):291-4.

20. Dolenc $V$. Treatment of fusiform aneurysms of the peripheral cerebral arteries. Report of two cases. J Neurosurg. 1978;49(2):272-7.

21. Chang HS, Fukushima T, Miyazaki S, Tamagawa T. Fusiform posterior cerebral artery aneurysm treated with excision and end-to-end anastomosis. Case report. J Neurosurg. 1986;64(3):501-4.

22. Vajkoczy P. Revival of extra-intracranial bypass surgery. Curr Opin Neurol. 2009;22(1):90-5.

23. Brockmann C, Gerigk L, Vajkoczy P, Groden C, NeumaierProbst $\mathrm{E}$. Magnetic resonance imaging flow quantification of non-occlusive excimer laser-assisted EC-IC high-flow bypass in the treatment of complex intracranial aneurysms. Clin Neuroradiol. 2012;22(1):39-45.

24. Ewald $\mathrm{CH}$, Kühne D, Hassler WE. Bypass-surgery and coilembolisation in the treatment of cerebral giant aneurysms. Acta Neurochir (Wien). 2000;142(7):731-7.

25. Seo BR, Kim TS, Joo SP, Lee JM, Jang JW, Lee JK, et al. Surgical strategies using cerebral revascularization in complex middle cerebral artery aneurysms. Clin Neurol Neurosurg. 2009;111(8):670-5.

26. Sanai N, Zador Z, Lawton MT. Bypass surgery for complex brain aneurysms: an assessment of intracranial-intracranial bypass. Neurosurgery. 2009;65(4):670-83.

27. Kan P, Liu JK, Couldwell WT. Giant fusiform aneurysm in an adolescent with PHACES syndrome treated with a high-flow external carotid artery-M3 bypass. Case report and review of the literature. J Neurosurg. 2007;106(Suppl 6):495-500.

28. Tulleken CA, van der Zwan A, van Rooij WJ, Ramos LM. High-flow bypass using nonocclusive excimer laser-assisted end-to-side anastomosis of the external carotid artery to the $\mathrm{P} 1$ segment of the posterior cerebral artery via the sylvian route. Technical note. J Neurosurg. 1998;88(5):925-7.

29. Streefkerk HJ, Wolfs JF, Sorteberg W, Sorteberg AG, Tulleken CA. The ELANA technique: constructing a high flow bypass using a non-occlusive anastomosis on the ICA and a conventional anastomosis on the SCA in the treatment of a fusiform giant basilar trunk aneurysm. Acta Neurochir (Wien). 2004;146(9):1009-19.

30. Thines L, Agid R, Dehdashti AR, da Costa L, Wallace $\mathrm{MC}$, Terbrugge KG, et al. Assessment of extracranialintracranial bypass patency with 64-slice multidetector computerized tomography angiography. Neuroradiology. 2009;51(8):505-15.

31. Elhammady MS, Wolfe SQ, Farhat H, Ali Aziz-Sultan M, Heros RC. Carotid artery sacrifice for unclippable and uncoilable aneurysms: endovascular occlusion vs common carotid artery ligation. Neurosurgery. 2010;67(5):1431-6.

32. Arat A, Islak C, Saatci I, Kocer N, Cekirge S. Endovascular parent artery occlusion in large-giant or fusiform distal posterior cerebral artery aneurysms. Neuroradiology. 2002;44(8):700-5.

33. Martin NAK, Coiteiro ID. Bypass techniques for the treatment of intracranial aneurysms. Operative Tech Neurosurg. 2000;3:255-70.
34. Onodera H, Hiramoto $\mathrm{J}$, Morishima H, Tanaka $\mathrm{Y}$, Hashimoto T. Treatment of an unruptured fusiform aneurysm of the internal carotid artery associated with Wegener's granulomatosis by endovascular balloon occlusion. Case report. Neurol Med Chir (Tokyo). 2012;52(4):216-8.

35. Murakami K, Shimizu H, Matsumoto Y, Tominaga T. Acute ischemic complications after therapeutic parent artery occlusion with revascularization for complex internal carotid artery aneurysms. Surg Neurol. 2009;71(4):434-41.

36. Suh SJ, Kim SC, Kang DG, Ryu KY, Lee HG, Cho JH. Clinical and angiographic results after treatment with combined clipping and wrapping technique for intracranial aneurysm. J Korean Neurosurg Soc. 2008;44(4):190-5.

37. Kato $\mathrm{Y}$, Sano H, Kanno T. Creation of an instant wrapping clip with GORE-TEX ${ }^{\circledast}$ sheet. J Clin Neurosci. 1999;4(4): 486-7.

38. Fujitsu K, Ishiwata Y, Gondo G, Fujii S, Feng DD. Wrapclipping with a Dacron mesh silastic sheet. Technical note. J Neurosurg. 1994;80(2):336-7.

39. Herrera O, Kawamura S, Yasui N, Yoshida Y. Histological changes in the rat common carotid artery induced by aneurysmal wrapping and coating materials. Neurol Med Chir (Tokyo). 1999;39(2):134-9.

40. Andres RH, Guzman R, Weis J, Schroth G, Barth A. Granuloma formation and occlusion of an unruptured aneurysm after wrapping. Acta Neurochir (Wien). 2007;149(9):953-8.

41. Kim YJ. Sole stenting technique for treatment of complex aneurysms. J Korean Neurosurg Soc. 2009;46(6):545-51.

42. Byrne JV, Beltechi R, Yarnold JA, Birks J, Kamran M. Early experience in the treatment of intra-cranial aneurysms by endovascular flow diversion: a multicentre prospective study. PLoS One. 2010;5(9).pii:e12492.

43. Crowley RW, Evans AJ, Kassell NF, Jensen ME, Dumont AS. Endovascular treatment of a fusiform basilar artery aneurysm using multiple "in-stent stents". Technical note. J Neurosurg Pediatr. 2009;3(6):496-500.

44. Higashida RT, Smith W, Gress D, Urwin R, Dowd CF, Balousek PA, et al. Intravascular stent and endovascular coil placement for a ruptured fusiform aneurysm of the basilar artery. Case report and review of the literature. $J$ Neurosurg. 1997;87(6):944-9.

45. Luo Q, Wang H, Xu K, Yu J. Endovascular treatments for distal posterior cerebral artery aneurysms. Turk Neurosurg. 2012;22(2):141-7.

46. Shapiro M, Becske T, Sahlein D, Babb J, Nelson PK. Stentsupported aneurysm coiling: a literature survey of treatment and follow-up. AJNR Am J Neuroradiol. 2012;33(1):159-63.

47. Jeong SM, Kang SH, Lee NJ, Lim DJ. Stent-assisted coil embolization for the proximal middle cerebral artery fusiform aneurysm. J Korean Neurosurg Soc. 2010;47(5):406-8.

48. Mawad ME, Cekirge S, Ciceri E, Saatci I. Endovascular treatment of giant and large intracranial aneurysms by using a combination of stent placement and liquid polymer injection. J Neurosurg. 2002;96(3):474-82.

\section{Endereço para correspondência}

Maria Luana Carvalho Viégas

Av. José Bonifácio, 2464, casa 5, Guamá

66065-362 - Belém, PA, Brasil

E-mail:mluanaviegas@gmail.com 